\title{
Separation of Stem Cells from Follicle, Pulp and Periodontal Ligament of Impacted Third Molar
}

\author{
Abbass Kamali ${ }^{1}$, Pooya Olyaee ${ }^{2}$, Fatemeh Rahbari Zadeh ${ }^{3}$, Seyyedeh Nazanin Al Ali ${ }^{4}$, Maryam Baghizadeh \\ Fini ${ }^{5,6 *}$ \\ ${ }^{1}$ Department of Laser, Faculty of Dentistry, Tehran University of Medical Sciences, Iran
}

${ }^{2}$ Department of Orthodontics, Urmia University of medical sciences, Iran

${ }^{3}$ Professor, Biotechnology department, Tarbiat Modarres University, Iran

${ }^{4}$ General Dentist, Tehran, Iran

${ }^{5}$ School of Dentistry, Tehran University of Medical Sciences, Tehran, Iran

${ }^{6}$ Master of Health Promotion, Oklahoma State University, Stillwater, USA

Submission: April 15, 2020; Published: April 22, 2020

*Corresponding author: Maryam Baghizadeh Fini, School of Dental, Tehran University of Medical Sciences \& Master of Health Promotion, Oklahoma State University, US

Abstract

Aim: The goal of this study is the separation and culture of stem cells, which are important in tissue engineering. This basic research was carried out to separate stem cells from different tissues of the third molar impacted teeth (Pulp, Periodontal ligament, Follicle).

Methods and Materials: Forty patients with good general health status, in an age range of 15 to 50 years were selected. The teeth were immediately placed in the transfer medium after performing the surgery. After two hours, different tissues were detached in the stem cell laboratory, and placed in the main culture medium. The main culture medium consisted of $80 \% \alpha$-Mem, and 20\% FCS (fetal calf serum). After three days, if stem cell attachment was observed, the cells were kept in 25 cc flasks (first passage). After 3-14 days, the trypsin enzyme was added to detach the cells. The dishes were checked until the culture medium was full of stem cells. Stem cell factor was used to inhibit the differentiation of stem cells. Upon separation, the cells were stored for a period of two weeks at a temperature of - $70 \mathrm{C}$ and subsequently at -196 C in the cell bank. In the next phase, stem cells were diagnosed and confirmed using a monoclonal magnetic antibody, and Real-Time gene expression.

Results: Stem cells of follicular tissue proliferated rapidly and attached to the culture medium in a short period of time. Stem cells of pulp proliferated and attached with some delay compared to follicular stem cells. The separation and proliferation of periodontal ligament stem cells were done successfully in just one of the samples.

Conclusion: Stem cells can be separated from different dental tissues and used in dental and maxillofacial reconstruction after culturing them.

Keywords: Stem cells, Dental pulp; Periodontal ligament; Dental follicle; Third molar; Impacted tooth

Abbreviation: PCR: Polymerase Chain Reaction; DPSC: Dental Pulp Stem Cells; PDLSC: Periodontal Ligament Stem Cells; PAFSC: Periapical Follicle Stem Cells; MBMSC: Mandibular Bone Marrow Stem Cells

\section{Introduction}

Stem cells are undifferentiated cells capable of proliferation, self-maintenance, production of different specialized cells, selfrepair of tissues from injury, and the capability to develop into a variety of cell types throughout the body. Stem cells replicate via self-renewal and these cells vary in their location and specialized functions [1]. The term "tissue engineering" refers to practical regeneration of the tissues missed or damaged due to infection, cancer, trauma, etc. Ivanoski et al. [2] published a review article on stem cell application in reconstructive treatments and tissue engineering, briefing opinions of world-known researchers [2]. Stem cells remodel body cells after pathosis and trauma. The potential ability of stem cells in tissue regeneration has led dental researchers to use them to treat different pathologies sustained by pulp and periodontium, and eventually regenerating the tooth itself. The term "cell therapy" in regenerative medicine is in this connection. To attain the therapeutic objective of treatment, stem 
cells or progenitor cells should be present at the site of damage to receive the inductive signals, stimulating their potential capability to proliferate and differentiate into the cells of the damaged tissues. These cells will regenerate the damaged tissue [3-7]. Therefore, for regenerative treatments in dentistry, one must intervene with proliferation to induction, and this is what tissue engineering deals with. There are crucial and delicate points to be concerned in this field since the separation of stem cells from connective tissue and its reproduction is a complicated process demanding experts and advanced biotechnological means.

Stem cells have three properties of self-renewal, clonogenicity, and undifferentiation [8]. In their classification, Evan et al have divided stem cells into embryonic and adult subgroups, indicating that embryonic cells have the potential of changing into any other cells. However, there are ethical restrictions resulting in embryonic mortality and morbidity $[3,4]$. This limitation is less prominent in adult stem cells, but the drawback is that the potential of these cells is limited to regenerating the same tissue; in other words, potential of each cell depends on the type of its original tissue. On this basis, Fortier announced that there are two types of adult stem cells, hematopoietic and mesenchymal [5]. An adult stem cell must create the cellular line where it resides and at the same time remains undifferentiated (self-renewal), this means organisms perpetuation. In different regenerative works, the researcher should separate stem cells of each tissue employing advanced techniques. Separation is carried out based on certain surface antigenic markers [4]. Surface antigenic markers are the characteristic of each cell and are coded by coding genes. Cellular diagnostic methods such as polymerase chain reaction (PCR), reverse transcription Polymerase chain reaction (RT-PCR), Realtime, Macro Array, and Laser Flow Cytometry have been used to detect the marker genes of the stem cells. For instance, in RT-PCR, mRNA is extracted from the target cells and after synthesis of cDNA (complementary DNA) and final product of PCR is studied using electrophoresis agar gel.

Some of the specific cellular markers of dental and periodontal stem cells, especially pulp, include DPP, DSP, Stro-1, CD44, CD149, VCA MI, Integrin B1, alkaline phosphatase.

The researcher extracts and confirms the stem cells on the basis of their surface markers and after repeated cultures in highly special media, a large number of stem cells are attained which can be used in extensive regenerations [9 -11]. Stem cell application in dentistry and other fields is under study. One of the pioneer researchers in this area is Professor Song Taoshi. In a series of research works since 2001, he separated the stem cells of pulp at NIH and regenerated the radicular and periodontal tissues of a rat at the dental faculty of USC. He separated stem cells of root apex papilla that are responsible for the root growth and PDL. He believes that in the near future, the wisdom tooth and deciduous teeth can be used to regenerate living human dental tissues [1215]. Ohazama et al. [16] separated pulpal stem cells of rat and transplanted the bud to the kidney capsule to attain dental and periodontal tissues [16]. Regarding the abovementioned, the first stage of this study was the separation of stem cells from the follicle, pulp, and periodontal ligament of the impacted third molar to follow the application after extraction and culture.

\section{Method and Materials}

To separate stem cells from the follicle, pulp, and periodontal ligament of the impacted third molar, three stages were carried out
a) Separation and culture
b) Diagnosis
c) Storage

\section{i. Stage one: Separation and Culture}

An age range of 15 to 45 years patients in good general health were selected and their impacted third molars were extracted observing all standard principles. Immediately after extraction, the teeth were laid in the first transfer medium designated - $\alpha$ Mem. This prepared initial culture medium consists of sugar, glutamine, special proteins, penicillin, and streptomycin. Unfortunately, fungal contamination caused the first ten samples to be excluded. Therefore, antifungal agents were added to the $\alpha$-Mem medium. The transfer medium was kept in cold condition and the samples were transferred to the laboratory in the shortest time possible after tooth extraction. The tissues were detached using sharp and sterile curettes in the laboratory, but the periodontal ligament was not detached mechanically (special centrifuge system) because of low cellular volume, and the process was done using collagenase enzyme and $3 \mathrm{mg}$ of dispase. After 30 minutes, the sample was passed through a mesh having 70-micrometer pores.

The detached cells were subsequently entered into the main culture medium. The main culture medium consisted of $80 \%$ $\alpha$-Mem, and 20\% FCS. The culture medium was in six-part plates. In this stage, the medium was placed in a $50 \% \mathrm{CO}_{2}$ incubator with a temperature of 37 degrees centigrade. After three days, the cultured samples were studied under a microscope. If stem cell attachment was observed, the previous culture medium was washed, and the cells were kept in 25 cc flasks. After 3-14 days, trypsin enzyme was added to detach the cells and since trypsin is toxic, $\alpha$-Mem was immediately added to neutralize it. During this time, the $25 \mathrm{cc}$ dishes were repeatedly checked under a microscope until the culture medium was full of stem cells. Stem cell factor was applied to inhibit the differentiation of stem cells. Ultimately, after separation, the cells were stored for a period of two weeks at a temperature of $-70 \mathrm{C}$ and subsequently at $-196 \mathrm{C}$ in the cell bank.

\section{ii. Stage two, and three: Diagnosis and Confirmation of Stem Cells}

Thirty samples from different tissues without any fungalmicrobial contamination and cultured successfully were studied for diagnosis and confirmation of stem cells as below 
a) Monoclonal magnetic antibody (MACS kit); by this method, stem cells were separated to a great extent, but this is not still confirmable.

b) To confirm with $100 \%$ accuracy, gene expression (similar to PCR) was applied, this method is known as Real-Time, which detects mRNA with high accuracy and confirms stem cell presence.

\section{Results}

Thirty samples separated from impacted third molars underwent precise laboratory processes. Tissues of pulp, periodontium, and follicle were supposed to be extracted. Stem cells of dental follicular tissue proliferated rapidly and attached to the culture medium in a short period of time. This finding was observed in all samples. Stem cells of dental pulp proliferated and attached with some delay compared to follicular stem cells. This was seen in more than two-thirds of the samples. Only in one of the samples, the separation and proliferation of periodontal ligament stem cells were done successfully, and this finding was not seen in other samples.

\section{Discussion}

Using mesenchymal stem cells is a new and promising method for regeneration of different tissues including maxillofacial tissues and teeth in particular. There is a general acceptance of different dental tissues as stem cell resources, owing to the capability of permanent regeneration of these tissues. The major problem happens in the clinical utilization of these resources. Separation and storage of stem cells as an autogenous cellular bank are not an easy task but still possible. This issue is more important in extensive regenerations. However, the application of allogeneic stem cells may be probable in the near future, but the most crucial issue in this regard is antigenicity and cross infection. Researchers declared the main objective of separating the stem cells, their application in favor of different extensive defects, and incurable pathologies. In fact, using stem cells provides a new approach to treat different defects and diseases that we are incapable of treating them with prevailing conventional methods. The first step is to find cells which have preserved most of their embryonic properties or so-called multipotent. In this field, umbilical cells of the neonate have been the topic of extensive research. However, it should be noted that stem cells of each tissue, responsible for its remodeling, are the best resources that can separate and reproduce employing special methods and culture media. A review of the literature shows that from 1960s researchers (e.g., Owen and Friedonstein) were able to separate the stem cells of bone marrow after birth and transplant to produce bone, cartilage, lipid, and blood stroma. They called these cells Bone Marrow Stromal Stem Cells (BMSCs) $[17,18]$.

Following this study, many other investigations were carried out and in the present decade, a burst of studies in this area is witnessed. Ivan (1981 and 1991) classified stem cells into embryonic and adult subgroups and recognized them having high potential to regenerate tissues [3,4]. Nemet [19] introduces criteria for identifying periodontal ligament stem cells [19]. Ivanoski [2] collected the information on stem cells, stating that stem cells have diagnostic and therapeutic potentials [2]. Extensive research work in the field of application of stem cells has been done in dentistry during recent years, particularly in NIH. Since 2001, Professor Shi was able to separate stem cells from dental tissues. He believes that in the near future, live dental implants will be placed in the patients' jaws [12-15]. Ozahama et al. [16] could regenerate the root of rat's tooth in the kidney capsule [16]. Dualibi MT et al. [20] managed to make dental bud in a special culture medium [20]. Robey et al. [21] used stem cells for reconstruction of the tooth and maxillofacial tissues [21].

Jo et al. [22] tested the presence of dental pulp stem cells (DPSC) and periodontal ligament stem cells (PDLSC). Additionally, periapical follicle stem cells (PAFSC) from the developing root of the impacted third molar and mandibular bone marrow stem cells (MBMSC) were investigated for their optimal growth conditions, proliferation rates, and differentiation capabilities. They compared the properties of various dental stem cells and showed that DPSC, PDLSC, PAFSC, and MBMSC are similar to other MSCs with respect to their expression of STRO-1. However, there are significant differences in stem cell properties depending on the tissue source. These results suggest the use of optimal dental tissue as a source of stem cells for developing bioengineered organs [22]. Choi et al. [22] investigated the stem cell properties of PDL cells by inducing chondrogenesis using growth factors, TGF-b3, and BMP-6. This work demonstrated the potential of dental stem cells as a valuable cell source for chondrogenesis, which can be applied to the regeneration of cartilage in the tissue engineering field. These results also confirm that PDLSCs can be used in tissue engineering approaches for the treatment of osteoarthritis, temporomandibular joint disorder, and cartilage reconstruction [23]. Research on the application of stem cells in dentistry is in process; in the present study, the interesting issue was using follicular stem cells. Researchers mention the umbilical cord as a source of multipotent cells capable of generating different tissues. A cell bank has been established for storing stem cells of the neonate's umbilical cord [24]. In this article, stem cells were separated from follicles owing to the fact that they have preserved their embryonic properties to a greater extent, which were witnessed in our culture media. They attached much sooner than the stem cells of other tissues, filling out the culture medium. This promises the possibility of using follicles instead of the umbilical cord as a source of stem cells for each individual. In addition to the regeneration of dental and periodontal tissues, these personal stem cells can be used for maxillofacial reconstruction.

\section{Acknowledgement}

i. Authors would like to express their gratitude to the personnel and academics of Research Center/ Tehran Faculty of Dentistry, Biotechnology department of Tarbiat Modarres University in Tehran, and in particular Stem Cells Organization. 


\section{References}

1. Caracappa JD, Gallicchio VS (2019) The future in dental medicine: Dental stem cells are a promising source for tooth and tissue engineering. J Stem Cell Res Ther 5(2): 30-36.

2. Ivanovski S, Gronthos S, Shi S, Bartold PM (2006) Stem cells in the periodontal ligament. Oral diseases 12(4): 358-363.

3. Evans MJ, Kaufman MH (1981) Establishment in culture of pluripotential cells from mouse embryos. Nature 292(5819): 154-156.

4. Evans GS, Potten CS (1991) Stem cells and the elixir of life. Bioessays 13(3): 135-138.

5. Fortier LA (2005) Stem cells: classifications, controversies, and clinical applications. Vet Surg 34(5): 415-423.

6. Narang S, Sehgal N (2012) Stem cells: A potential regenerative future in dentistry. Indian J hum genet 18(2): 150-154.

7. Chalisserry EP, Nam SY, Park SH, Anil S (2017) Therapeutic potential of dental stem cells. Journal of tissue engineering 8: 2041731417702531.

8. Pamela G, Bianco P (2006) The use of adult stem cells in rebuilding the human face. JADA 137(7): 961-972.

9. Hargreaves KM, Goodis HE, Tay FR (2012) Seltzer and Bender's dental pulp. Chicago: Quintessence.

10. Miura M, Gronthos S, Zhao M, Lu B, Fisher LW, et al. (2003) SHED: stem cells from human exfoliated deciduous teeth. Proceedings of the National Academy of Sciences 100(10): 5807-5812.

11. Mezey É, Key S, Vogelsang G, Szalayova I, Lange GD, et al. (2003) Transplanted bone marrow generates new neurons in human brains. Proc Natl Acad Sci 100(3): 1364-1369.

12. Shi S, Robey PG, Gronthos S (2001) Comparison of human dental pulp and bone marrow stromal stem cells by cDNA microarray analysis. Bone 29(6): 532-539.

13. Shi S, Gronthos S, Chen S, Reddi A, Counter CM, et al. (2002) Bone formation by human postnatal bone marrow stromal stem cells is enhanced by telomerase expression. Nature biotechnology 20(6): 587591
14. Shi S, Gronthos S (2003) Perivascular niche of postnatal mesenchymal stem cells in human bone marrow and dental pulp. J bone miner res 18(4): 696-704.

15. Shi S, Bartold PM, Miura M, Seo BM, Robey PG, et al. (2005) The efficacy of mesenchymal stem cells to regenerate and repair dental structures. Orthod craniofac res 8(3): 191-199.

16. Ohazama A, Modino SA, Miletich I, Sharpe PT (2004) Stem-cell-based tissue engineering of murine teeth. Journal of dental research 83(7): 518-522.

17. Friedenstein AJ, Piatetzky-Shapiro II, Petrakova KV (1966) Osteogenesis in transplants of bone marrow cells. J Embryol Exp Morphol 16(3): 381-390.

18. Owen M, Friedenstein AJ (1988) Stromal stem cells: marrow-derived osteogenic precursors. In Ciba Found Symp 136(29): 42-60.

19. Nemeth E, Kulkarni GW, McCulloch CA (1993) Disturbances of gingival fibroblast population homeostasis due to experimentally induced inflammation in the cynomolgus monkey (Macaca fascicularis): potential mechanism of disease progression. Journal of periodontal research 28(3): 180-191.

20. Duailibi MT, Duailibi SE, Young CS, Bartlett JD, Vacanti JP, et al, (2004) Bioengineered teeth from cultured rat tooth bud cells. J dent res 83(7): 523-528.

21. Robey PG (2005) Post-natal stem cells for dental and craniofacial repair. Oral Biosci Med 2(2/3): 83-90.

22. Jo YY, Lee HJ, Kook SY, Choung HW, Choung PH, et al. (2007) Isolation and characterization of postnatal stem cells from human dental tissues. Tissue engineering 13(4): 767-773.

23. Choi S, Cho TJ, Kwon SK, Lee G, Cho J (2013) Chondrogenesis of periodontal ligament stem cells by transforming growth factor- $\beta 3$ and bone morphogenetic protein- 6 in a normal healthy impacted third molar. International Journal of Oral Science 5(1): 7-13.

24. Erices A, Conget P, Minguell JJ (2000) Mesenchymal progenitor cells in human umbilical cord blood. British Journal of Haematology 109(1): 235-242.
This work is licensed under Creative Commons Attribution 4.0 License DOI: 10.19080/ADOH.2020.12.555839 